\title{
Correction to: Extraction of Leaf-Vein Parameters and Classification of Plants Using Machine Learning
}

\author{
Guruprasad Samanta, Amlan Chakrabarti, and Bhargab \\ B. Bhattacharya
}

\section{Correction to: chapter "Extraction of Leaf-Vein Parameters and Classification of Plants Using Machine Learning" in: D. Bhattacharjee et al. (eds.), Proceedings of International Conference on Frontiers in Computing and Systems, Advances in Intelligent Systems and Computing 1255, https://doi.org/10.1007/978-981-15-7834-2_54}

The original version of the book was inadvertently published without few lines in the conclusion part in Chapter 54. This correction chapter is updated with the missing lines as follows:

\section{Conclusion}

We have studied for the first time, some graph-theoretic features present in leaf-vein networks of three trees of the Indian subcontinent. Based on these features, data clustering and identification have been performed using $K$-means and $K N N$ algorithms, respectively. Our study reveals that among a database of 30 images, 26 samples could be properly classified with an accuracy of $86 \%$. Some misclassification was noted between Jackfruit and Peepal samples whose vein structures are close to each other with respect to the above feature-set. On a positive side, only

The online version of this chapter can be found at https://doi.org/10.1007/978-981-15-7834-2_54 
two instances of misclassification were observed between Peepal and Mango. To the best of our knowledge, no similar work on leave-venation of Indian trees has been reported in the literature, and hence no comparison could be made with the previous approaches or any other state-of-the-art approaches. The proposed methodology is simple; we may need to consider more features in order to classify an unknown sample correctly from a larger class of different leaves. The analysis of leaf-vein networks may help in understanding the interplay between vein architecture and photosynthesis. For future studies, it would be interesting to explore new methods based on computational geometry or deep neural network and to identify the structural hierarchy present in the venation network for various types of leaves from different types of plants. Further investigation in this direction would also enhance our understanding of genotype and phenotype of plant species and various ecological parameters that determine the leaf-venation patterns. 